\title{
Método da Procura em Rede Melhorado: uma Proposta para a Estimação dos Parâmetros do Modelo de Rakhmatov e Vrudhula
}

\author{
B.F. SILVA, A. SAUSEN*, P.S. SAUSEN e M. REIMBOLD
}

Recebido em 29 maio, 2013 / Aceito em 22 dezembro, 2013

\begin{abstract}
RESUMO. Neste artigo é apresentada a proposição de um novo método de otimização denominado Procura em Rede Melhorado, que é uma extensão do método Procura em Rede Modificado, aplicado no cálculo dos parâmetros empíricos do modelo de Rakhmatov e Vrudhula, utilizado na literatura para a predição do tempo de vida de baterias usadas em dispositivos móveis. O novo método foi avaliado considerando a seguinte metodologia, inicialmente os parâmetros empíricos do modelo foram calculados considerando os métodos de otimização Procura em Rede Melhorado, Procura em Rede Modificado e Mínimos Quadrados, assim como os dados experimentais obtidos a partir de uma plataforma de testes, considerando uma bateria de Lhitium-Íon, modelo BL5F, usada no telefone celular Nokia N95. Em um segundo momento, o modelo de Rakhmatov e Vrudhula foi validado para cada conjunto de parâmetros obtidos, e os dados simulados, a partir do modelo, foram comparados com dados experimentais adquiridos da plataforma testes. Considerando os resultados das simulações foi realizada uma análise comparativa e verificou-se que com a aplicação do método Procura em Rede Melhorado, na estimação de parâmetros do modelo de Rakhmatov e Vrudhula, foi possível obter uma implementação fácil e intuitiva, melhorar a acurácia do modelo, bem como preservar o tempo de execução.
\end{abstract}

Palavras-chave: estimação de parâmetros, Procura em Rede Melhorado, modelo RV.

\section{INTRODUÇÃO}

Atualmente, a evolução tecnológica permite acessar informações em qualquer lugar do planeta, a qualquer momento. Esta facilidade é garantida, principalmente, através da utilização da tecnologia de comunicação sem fio a partir do uso de dispositivos móveis, nas mais diversas áreas. São exemplos destes dispositivos móveis: telefones celulares, câmeras digitais, tablets, notebooks, entre outros. A tendência de mercado é haver dispositivos móveis menores em tamanho, cada vez mais velozes, que desempenham as mais variadas funções, conectados a uma rede mundial de serviços e recursos, através de uma infraestrutura de alto desempenho.

\footnotetext{
* Autor correspondente: Airam Sausen

Programa de Pós-Graduação Stricto Sensu em Modelagem Matemática, Departamento de Ciências Exatas e Engenharias, DCEEng, Universidade Regional do Noroeste do Estado do Rio Grande do Sul, UNIJUÍ, 98700-000 Ijuí, RS, Brasil. E-mails: bolivarfernandes@gmail.com; airam@unijui.edu.br; sausen@unijui.edu.br; manolo@unijui.edu.br
} 
Os dispositivos móveis são mantidos energeticamente por algum tipo de bateria recarregável, que são limitadas em tamanho e, portanto em capacidade. Deste modo, o uso destes aparelhos está condicionado ao tempo de vida da bateria, que é por definição o tempo que a mesma demora para atingir o nível mínimo de capacidade de carga, denominado nível de cutoff [21]. Quando a bateria alcança o nível de cutoff as reações eletroquímicas cessam, neste momento a bateria deixa de fornecer energia ao sistema sendo considerada descarregada.

Portanto, no projeto de dispositivos móveis o tempo de vida da bateria é considerado uma das características mais importantes, pois informa o tempo que o dispositivo poderá ser utilizado sem a necessidade de conectá-lo a uma fonte externa. Desta forma, é importante dispor de algum método que seja capaz de predizer o tempo de vida da bateria, e assim definir o funcionamento de todo sistema que é alimentado por ela. Uma das formas é através de modelos matemáticos que descrevem a descarga de energia de uma bateria. Nos últimos anos diferentes modelos matemáticos foram desenvolvidos, dentre eles é possível citar: os analíticos [11, 13, 17, 20, 21, 22, 23], os estocásticos [8], os eletroquímicos [11], e os elétricos [7, 18, 19], cada um com suas características e níveis de complexidade. Considerando os trabalhos correlatos observa-se que os modelos que apresentam os melhores resultados na predição do tempo de vida de baterias são aqueles que consideram os efeitos não lineares que ocorrem nas operações de descarga [20].

No trabalho de Oliveira [17] é apresentado um estudo comparativo entre três modelos analíticos da literatura utilizados para a predição do tempo de vida de baterias: o modelo Linear [10], a lei de Peukert [10] e o modelo analítico de difusão de Rakhmatov e Vrudhula, denominado neste trabalho de modelo RV [20]. Para a comparação destes modelos foram utilizados perfis de descargas constantes e perfis de descarga variáveis, obtidos a partir de uma plataforma de testes [16]. Nesta análise comparativa os modelos foram implementados na ferramenta computacional Matlab considerando os parâmetros de uma bateria de Lithium-Íon, modelo $B L 5 F$, utilizada no telefone celular Nokia N95. A partir dos resultados das simulações foi encontrado que o modelo RV é o mais acurado, quando comparado com os demais modelos simulados, com erro médio na predição do tempo de vida da bateria de 5,71\% para descargas constantes, e 6,5\% para descargas variáveis.

Considerando o modelo RV destaca-se que o mesmo considera as características não lineares que ocorrem em uma bateria tais como o efeito de recuperação e a taxa de capacidade. Neste modelo as principais propriedades da bateria são descritas a partir da Lei de Fick, onde o processo de difusão é representado por uma Equação Diferencial Parcial (EDP) com condições de fronteira de segunda espécie [20]. Observa-se que o modelo RV possui dois parâmetros empíricos, que dependem da bateria utilizada, e portanto precisam ser estimados, o $\alpha$ que está relacionado com a capacidade da bateria, e o $\beta$ que está relacionado ao comportamento não linear da bateria.

A estimação de parâmetros desconhecidos, em um modelo matemático, a partir de dados experimentais constitui um Problema Inverso (PI). Segundo a literatura [4, 6, 15, 24, 26] os PIs podem surgir de duas formas: a primeira consiste em um problema de reconstrução, ou seja, a partir do efeito (dados experimentais medidos ou observados) e de um modelo matemático (i.e., Problema Direto (PD)), busca-se uma causa desconhecida; e a segunda consiste em um problema de identi- 
ficação, ou seja, conhecidas as causas e os efeitos, busca-se encontrar os parâmetros do modelo. Neste artigo o PI considerado é um problema de identificação.

Nos trabalhos que utilizam o modelo RV, para a estimação dos seus parâmetros empíricos, verificou-se que todos eles usam o método de otimização dos Mínimos Quadrados (MQ) em batelada, isto é os parâmetros são estimados de uma única vez [17, 20, 21, 22, 23]. Este método consiste na procura do melhor ajuste para um conjunto de dados tentando minimizar a soma dos quadrados das diferenças entre o valor simulado pelo modelo e os dados experimentas, obtidos de uma plataforma de testes, de forma a maximizar o grau de ajuste do modelo em relação aos dados simulados.

Neste contexto, o principal objetivo deste artigo é a resolução do modelo RV para o cálculo do tempo de vida de baterias, considerando a proposição de um novo método de otimização denominado Método da Procura em Rede Melhorado (PRMe), que é uma extensão do método da Procura em Rede Modificado (PRM) proposto por Cervi \& Borges [3, 5]. Este novo método foi desenvolvido para ser aplicado ao problema de estimação de parâmetros associado ao modelo RV, objetivando fácil implementação, melhor precisão e boa velocidade do modelo.

O restante deste artigo está organizado como segue. Na Seção 2 são apresentadas as principais propriedades e características de uma bateria, em seguida é realizada uma breve revisão do estado da arte de modelos matemáticos para predição do tempo de vida de baterias utilizadas em dispositivos móveis, e por fim são apresentados os conceitos de PD e PI, bem como alguns métodos de otimização da literatura para a estimação de parâmetros, e usados neste trabalho para a comparação com o método proposto. Na Seção 3 é realizada a proposição do método de estimação de parâmetros PRMe. Na Seção 4 é apresentada a modelagem matemática. Na Seção 5 são apresentados os resultados das simulações e sua análise. E por fim na Seção 6 são apresentadas as conclusões, bem como as sugestões de trabalhos futuros.

\section{ESTADO DA ARTE}

Nesta seção inicialmente é realizada uma revisão bibliográfica sobre baterias, onde é abordado os principais tipos de baterias, suas propriedades e características não lineares. Em um segundo momento é realizada uma revisão bibliográfica dos modelos matemáticos encontrados na literatura para predição do tempo de vida de baterias usadas em dispositivos móveis. Por fim, são apresentados os conceitos de PD e PI, assim como alguns métodos de otimização da literatura que são usados neste trabalho para a comparação com o método proposto para estimação dos parâmetros do modelo RV.

\subsection{Baterias}

Uma bateria é constituída por uma ou mais células eletroquímicas, que podem ser conectadas em série, em paralelo, ou ainda através de uma combinação de ambas. Nestas células a energia química armazenada é convertida em energia elétrica através de reações eletroquímicas. Uma célula eletroquímica é formada por dois eletrodos: um ânodo que possui polaridade negativa, e 
um cátodo que possui polaridade positiva, separados por um eletrólito. Durante a fase de descarga (i.e., quando a bateria fornece energia elétrica a um circuito externo) o ânodo libera elétrons para o circuito, enquanto o cátodo recebe elétrons do circuito. Já na fase de carregamento da bateria, os processos químicos são revertidos. Os elétrons têm origem a partir de reações eletroquímicas e são chamados de espécies eletroativas.

Ao comparar diferentes tecnologias de bateria, surgem várias considerações. Estas incluem densidade de energia (carga armazenada por unidade de peso da bateria), ciclo de vida (o número de ciclos de carga/descarga antes do descarte da bateria), impacto ambiental, segurança, custo, alimentação de tensão disponível e características de carga/descarga. Os principais tipos de baterias recarregáveis, para aparelhos eletrônicos portáteis, são descritas por Jongerden [10], entre as existentes destacam-se: Níquel Cádmio (Ni-Cd), Níquel Metal-Hidreto (Ni-MH), Lithium-Íon (Li-Íon), Lithium Polímero e Alcalina Recarregável. A bateria utilizada neste trabalho é do tipo Li-Íon que é a tecnologia que mais cresce atualmente, com densidade de energia significativamente superior, e ciclo de vida aproximadamente duas vezes maior quando comparada as baterias de Ni-MH. As baterias de Li-Íon são mais sensíveis às características da corrente de descarga, e frequentemente equipam notebooks, tablets e telefones celulares.

Uma bateria possui duas importantes propriedades, que são: (i) a voltagem, medida em Volts $(V)$; e (ii) a capacidade, geralmente medida em Ampère-Hora $(A h)$, o produto destas duas grandezas fornece a quantidade de energia armazenada na bateria. Por exemplo, teoricamente, uma bateria de $100 A h$ é projetada para fornecer $5 A$ por 20 horas, ou então, $100 A$ durante 1 hora. Porém, esta representação é teórica, na prática, geralmente, a corrente de descarga não é constante no tempo, ou seja, as operações de descarga têm características não lineares, que influenciam diretamente no tempo de vida da bateria.

A modelagem do comportamento de baterias pode se tornar complexa se forem considerados os efeitos não lineares que ocorrem durante o período de descarga. No caso ideal, a tensão permanece constante durante todo o período de descarga, tornando-se zero quando a bateria está descarregada. O ideal seria que a capacidade fosse constante para qualquer corrente de descarga, e que toda a energia armazenada na bateria fosse utilizada. Contudo, em um procedimento de descarga de uma bateria real, a tensão é reduzida lentamente e a capacidade efetiva é reduzida para altas correntes. Ressalta-se que, dependendo do tipo de bateria, estes efeitos têm maiores ou menores consequências na sua capacidade [10].

As baterias possuem duas características não lineares importantes. A primeira delas é o efeito de recuperação [10, 20, 21, 22], que é definido pela reorganização dos elétrons no eletrólito durante um período de relaxação, i.e. intervalo de tempo em que a corrente de descarga é reduzida significativamente. Neste intervalo os elétrons se reorganizam de maneira uniforme, de modo que o sistema recupere o equilíbrio e o gradiente de concentração seja nulo no eletrólito. Assim, a capacidade efetiva da bateria é aumentada, pois uma maior quantidade de carga torna-se disponível antes do sistema alcançar o nível de cutoff. Já a segunda característica é o efeito da taxa de capacidade que depende da capacidade atual da bateria e da intensidade da corrente de descarga, ou seja, com uma alta corrente de descarga a capacidade efetiva da bateria é baixa, pois não 
há tempo suficiente para a reorganização dos elétrons no eletrólito (i.e., efeito de recuperação), assim mais carga permanece sem ser utilizada pelo sistema, reduzindo a capacidade e o tempo de vida da bateria. Já com correntes alternadas, a capacidade efetivada da bateria é aumentada, pois na troca de uma corrente alta para uma corrente baixa, ou até mesmo para um período sem corrente, ocorre a reorganização dos elétrons no eletrólito, tornando disponível uma maior quantidade de carga na superfície do eletrodo, aumentando a capacidade efetiva da bateria. Desta maneira, quanto maior a quantidade de carga presente no eletrólito no momento da relaxação, mais carga será possível recuperar, e consequentemente estará disponível na superfície do eletrodo para ser utilizada pelo sistema. Ressalta-se que esta disponibilidade de carga na superfície do eletrodo nunca será maior, nem igual à inicial.

\subsection{Modelos de Baterias}

Existem diferentes modelos matemáticos que são utilizados em projetos de sistemas alimentados por baterias, pois permitem a análise do comportamento de carga e descarga sob diferentes especificações de projeto. Nesta seção é realizada uma descrição dos principais modelos matemáticos encontrados na literatura que realizam esta função.

Os modelos eletroquímicos [11] baseiam-se nos processos químicos que ocorrem na bateria e por isso são descritos com significado nível de detalhamento. Isto faz com que tais modelos sejam considerados mais acurados. No entanto, sua descrição detalhada torna-os altamente complexos e difíceis de implementar, devido à grande quantidade de parâmetros da bateria que devem ser definidos pelo usuário.

Os modelos elétricos [7, 18, 19], também denominados de modelos de circuitos elétricos, empregam uma combinação de fontes de tensão, resistores e capacitores para a simulação da descarga da bateria, também consideram a taxa de capacidade e os efeitos térmicos da bateria. Esta classe apresenta simulação de fácil entendimento, realizadas a partir da utilização de simuladores de circuito. Estes modelos, em decorrência de sua facilidade de uso e compreensão, têm sido utilizados amplamente, por engenheiros eletricistas, na análise de várias tecnologias de baterias [18].

Os modelos estocásticos [8] descrevem a bateria em um nível mais elevado de abstração do que os modelos eletroquímicos e elétricos. O processo de descarga é descrito a partir de processos estocásticos. Uma das desvantagens, desta classe de modelos, é que na sua grande maioria não consideram os efeitos não lineares presentes em uma descarga.

Os modelos analíticos [10, 17, 20, 21, 22, 23] descrevem a bateria de uma forma abstrata e as principais propriedades, i.e., o efeito da taxa de capacidade e o efeito de recuperação, são modeladas utilizando um conjunto reduzido de equações. Esta característica torna, este tipo de modelo, de fácil implementação computacional quando comparado aos modelos eletroquímicos e elétricos. Dentre os principais modelos analíticos encontrados na literatura destaca-se o modelo RV, descrito na Seção 4 e utilizado neste trabalho para a simulação do tempo de vida de baterias utilizadas em dispositivos móveis. 


\subsection{Métodos para Estimação de Parâmetros}

Nesta seção é apresentada uma breve revisão bibliográfica de alguns métodos de otimização, presentes na literatura, utilizados para estimação dos parâmetros empíricos do modelo RV. Para o entendimento dos métodos, inicialmente, são definidos os conceitos de PD e PI a partir da ilustração apresentada na Figura 1.

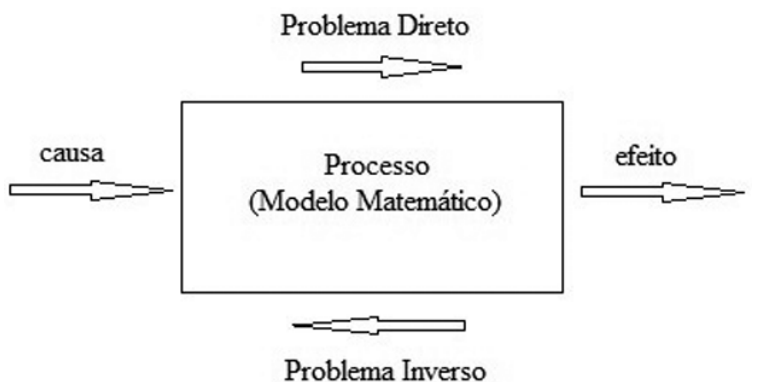

Figura 1: Representação esquemática do PD e do PI [6, 25].

Um PD consiste no conhecimento preciso das causas para determinação dos efeitos, que são as propriedades calculadas a partir de um modelo matemático, tais como: a temperatura, o tempo de vida, a corrente elétrica, a velocidade, entre outras. Por outro lado, um PI pode ser definido de duas formas: (i) a primeira, consiste na determinação das causas desconhecidas a partir de efeitos medidos ou observados, define-se como causas em um modelo matemático as condições iniciais, as condições de contorno, os termos de fonte, nestes casos o PI é um problema de reconstrução; (ii) a segunda, consiste na determinação das propriedades dos sistemas (i.e., parâmetros) a partir das causas e dos efeitos medidos ou observados, neste caso o PI é um problema de identificação $[6,9,15,14,24,25,26]$.

A seguir é apresentada uma breve descrição dos métodos de otimização utilizados neste trabalho para comparação com o método proposto.

\subsubsection{Método dos Mínimos Quadrados}

O método dos Mínimos Quadrados (MQ) consiste na procura do melhor ajuste para um conjunto de dados tentando minimizar a soma dos quadrados das diferenças entre o valor obtido pelo modelo e os dados observados. Segue uma descrição geral do método, seja um vetor $\zeta$ que contém os parâmetros $\theta_{n}$ a serem estimados, dado por

$$
\zeta=\left\{\theta_{1}, \ldots, \theta_{n}\right\}^{T}
$$

e o vetor $Z$ que contém as entradas $u(N)$ e as saídas $y(N)$ (i.e., medidas experimentais) ao longo de um intervalo de tempo $1 \leq t \leq N$ dado por

$$
Z^{N}=\{u(1), y(1), \ldots u(N), y(N)\} .
$$


O método MQ consiste em minimizar uma função objetivo dada por

$$
V_{N}\left(\vec{\zeta}, Z^{N}\right)=\frac{1}{N} \sum_{t=1}^{N}\left(y(t)-\hat{y}(t \mid \zeta)^{2} .\right.
$$

onde $y(t)$ são as saídas experimentais medidas, $\hat{y}(t \mid \zeta)$ são as saídas calculadas a partir do modelo que contém os parâmetros que se deseja estimar, $N$ é o número de dados experimentais $[1,12]$.

\subsubsection{Método Procura em Rede Modificado}

O método Procura em Rede Modificado (PRM) é uma extensão do método PR [24]. Este método foi proposto por Cervi e Borges [3,5] e consiste em definir para cada parâmetro a ser estimado um intervalo com um valor mínimo e máximo que possivelmente contenha um valor ótimo do parâmetro considerado, construindo assim uma rede de intervalos. De posse desta rede o PD é resolvido com todas as combinações dos valores que compõem a rede fazendo a busca pelo menor erro, entende-se por menor erro a menor diferença entre o valor obtido pelo cálculo do PD e o valor coletado experimentalmente.

A modificação deste método em relação ao método PR consiste em realizar uma nova procura a partir dos dados obtidos, efetuando uma partição dos intervalos iniciais a fim de construir uma nova rede, diminuindo os valores máximos e mínimos fazendo uma busca cada vez mais local. Objetivando refinar o valor ótimo este processo é repetido até que o critério de parada seja satisfeito. Mesmo que a modificação apresente um ganho em acurácia e precisão, o método continua sendo considerado de solução sub-ótima. Para a implementação do algoritmo do método PRM os seguintes passos devem ser seguidos:

1 Passo: Estima-se intervalos $I_{p}=\left[\beta_{p \min }, \beta_{p \max }\right]$ de valores para cada parâmetro $\beta_{p}$ onde $p=1,2,3, \ldots, n p$ (número de parâmetros), que contêm o valor ótimo de $\beta_{p}\left(\beta_{o t}\right)$.

$2^{\circ}$ Passo: Constrói-se uma partição de $n c$ dados $\beta_{p f}=\beta_{p \min }+(f-1) \Delta \beta_{p}$ com $f=$ $1,2,3, \ldots, n c$ e $\Delta \beta_{p}=\frac{\left(\beta_{p \max }-\beta_{p \min }\right)}{(n c-1)}$.

$3^{\circ}$ Passo: Para cada sequência de valores $\left(\beta_{1 f}, \beta_{2 f}, \ldots, \beta_{n p f}\right)$ com $f=1,2,3, \ldots, n c$ resolve-se o PD.

$4^{\circ}$ Passo: Calcula-se as diferenças $d_{i}$ entre as soluções estimadas e os dados experimentais utilizando a equação (2.2) dada por

$$
d_{i}=\sum_{j=1}^{z f} \sum_{t=0}^{t f}\left|F_{e s t}(j, t)-F_{\exp }(j, t)\right|^{2} .
$$

$5^{\circ}$ Passo: Identifica-se o menor valor de $d_{i}\left(d_{\min }\right)$. Esta diferença corresponde ao conjunto de parâmetros $\beta_{o t}$ para para o intervalo $I_{p}$.

$6^{\circ}$ Passo: Refinamento da solução. Defini-se um novo intervalo $I_{p}=\left[\beta_{p \min }, \beta_{p \max }\right]$, tal que $\beta_{p \min }=\beta_{p o t}-\Delta \beta_{p}$ e $\beta_{p \max }=\beta_{p o t}+\Delta \beta_{p}$. 
$7^{\circ}$ Passo: Repete-se os passos 2 a 6 , estimando tantos intervalos $I_{p m}, m=1,2,3, \ldots, n r$ (número de refinamentos) até que

$$
\left|d_{\min }^{i+1}-d_{\min }^{i}\right|<\varepsilon \quad \text { ou } \quad n r=n r_{o t},
$$

onde $\varepsilon$ e $n r_{o t}$ são critérios de parada.

A seguir é apresentado o método de otimização PRMe, proposto a partir de uma modificação do método PRM, para a estimação dos parâmetros empíricos $\alpha$ e $\beta$ do modelo RV, a partir de dados experimentais obtidos de uma plataforma de testes.

\section{MÉTODO DA PROCURA EM REDE MELHORADO}

Durante o estudo e implementação do método PRM verificou-se que apesar da sua simplicidade, o mesmo possui uma limitação, por ser um método de procura sub-ótimo o parâmetro desejado obrigatoriamente deve estar dentro do intervalo pré-definido, caso não se tenha um conhecimento prévio deste valor, o intervalo deve ser grande o suficiente de modo a conter o valor procurado, nestes casos a procura dos parâmetros pode se tornar um processo um tanto demorado.

Neste contexto, a modificação realizada, que gerou o método PRMe, tem por objetivo eliminar a condição de que o parâmetro precisa obrigatoriamente estar dentro de um intervalo considerado, de modo que a partir de um valor inicial para cada parâmetro a ser estimado seja criado um intervalo, de posse dos intervalos é construída uma rede que busca o melhor valor para cada parâmetro. Caso os parâmetros não estejam contidos nos intervalos inicialmente determinados, este processo é refeito a partir do melhor parâmetro encontrado na primeira iteração que será tomado como um novo valor inicial. Observa-se que no início da simulação é estabelecido um critério de convergência, de modo que a partir de qualquer valor inicial haverá um direcionamento das redes até encontrar o valor ótimo para cada parâmetro. Segue a descrição do método.

\subsection{Descrição do Método}

$1^{\circ}$ Passo: Determina-se valores iniciais $\theta_{1}, \theta_{2}, \cdots \theta_{n}$, para cada parâmetro que será estimado, onde $n$ é o número de parâmetros.

$2^{\circ}$ Passo: Forma-se um intervalo

$$
\operatorname{Int}_{n}=\left[\theta_{n} \text { inf }, \ldots, \theta_{n} \sup \right]
$$

para cada parâmetro a partir do seu valor inicial $\theta_{n}$ e da escolha de uma taxa unitária para $\rho$, que irá determinar a amplitude dos intervalos considerados. O limite inferior do intervalo $I n t_{n}$ é dado por

$$
\theta_{n \text { inf }}=\theta_{n}-\rho \theta_{n},
$$

e o limite superior do intervalo é dado por

$$
\theta_{n \text { sup }}=\theta_{n}+\rho \theta_{n}
$$


$3^{\circ}$ Passo: Constrói-se uma partição de pontos nos intervalos $I n t_{n}$, onde a quantidade de pontos $q$ define o tamanho do espaçamento $\lambda$, que é dado por

$$
\lambda=\frac{\left(\theta_{n \text { sup }}-\theta_{n \text { inf }}\right)}{q-1},
$$

assim cada intervalo é reescrito na seguinte forma

$$
\operatorname{Int}_{n}=\left[\theta_{n} \mathrm{inf}, \theta_{n} \mathrm{inf}+\lambda, \theta_{n} \mathrm{inf}+2 \lambda, \ldots, \theta_{n} \inf +(q-1) \lambda, \theta_{n} \text { sup }\right] .
$$

$4^{\circ}$ Passo: Resolve-se o PD considerando todas as combinações dos parâmetros contidos nos intervalos.

$5^{\circ}$ Passo: Calcula-se a diferença $d_{i}$ entre as soluções estimadas $S_{\text {est }}$ e os dados experimentais $S_{\text {exp }}$ para cada combinação de parâmetros utilizando a equação

$$
d_{i}=\sum_{i=1}^{N}\left|S_{\exp _{i}}-S_{e s t}\right|^{2},
$$

onde $N$ é o número de dados experimentais.

$6^{\circ}$ Passo: Identifica-se o menor valor de $d_{i}\left(d_{\min }\right)$. Este valor corresponde ao conjunto de parâmetros $\theta_{o t}$ que foram localizados nos intervalos $I_{n} t_{n}$ construídos a partir dos valores iniciais propostos $\theta_{1}, \theta_{2}, \cdots, \theta_{n}$.

$7^{\circ}$ Passo: De posse de $\theta_{n, o t}$ verifica-se se a diferença entre os valores experimentais e os valores calculados é menor que um erro $(\varepsilon)$ pré-estabelecido, se sim, os valores para os parâmetros foram encontrados.

Caso contrário, utiliza-se os seguintes critérios de parada

$$
\left|d_{\min }^{i+1}-d_{\min }^{i}\right|<\xi \quad \text { ou } \quad n r=n i_{o t}
$$

onde $\xi$ e $n i_{o t}$ (número de iterações) são determinados pelo usuário e então os passos de 2 a 6 são repetidos até o erro ser satisfeito.

Na próxima seção é apresentada a modelagem matemática do PD, i.e. o modelo RV, e do PI.

\section{MODELAGEM MATEMÁTICA}

\subsection{Problema Direto}

Nesta seção é apresentado o modelo RV que entre outras características considera os efeitos não lineares que ocorrem em baterias. O processo de difusão unidimensional da concentração é descrito pela lei de Fick

$$
-J(x, t)=D \frac{\partial C}{\partial x}(x, t)
$$


e pela EDP dada por

$$
\frac{\partial C}{\partial t}(x, t)=D \frac{\partial^{2} C}{\partial x^{2}}(x, t)
$$

onde $J(x, t)$ é o fluxo das espécies eletroativas em função do tempo $t$ e em função de uma distância $x$ do eletrodo, $D$ é a constante de difusão. Seja $C(x, t)$ a concentração de espécies eletroativas no tempo $t \in[0, L]$ e na distância $x \in[0, w]$ do eletrodo. Considerando uma bateria completamente carregada (i.e., $t=0$ ), a concentração de espécies é constante através do comprimento do eletrólito, então tem-se a seguinte condição inicial

$$
C(x, 0)=C^{*} .
$$

A bateria é considerada descarregada quando $C(0, t)$ é inferior ao nível de cutoff. De acordo com a lei de Faraday o fluxo da superfície do eletrodo $(x=0)$ é proporcional à corrente $i(t)$ (i.e., carga externa aplicada), e o fluxo na outra extremidade da região de difusão $(x=w)$ é zero. Estas proposições fornecem as seguintes condições de fronteira

$$
\begin{gathered}
\left.D \frac{\partial C}{\partial x}(x, t)\right|_{x=0}=\frac{i(t)}{v F A}, \\
\left.D \frac{\partial C}{\partial x}(x, t)\right|_{x=w}=0,
\end{gathered}
$$

onde $A$ é a área da superfície do eletrodo, $F$ é a constante de Faraday e $v$ é o número de elétrons envolvidos na reação eletroquímica na superfície do eletrodo.

Em Oliveira [17] é realizada a resolução da equação (4.2) com condição inicial dada pela equação (4.3) e condições de fronteira apresentadas nas equações (4.4) e (4.5) utilizando transformada de Laplace e transformada de Laplace Inversa obtendo-se a equação

$$
\alpha=\int_{0}^{L} \frac{i(\tau)}{\sqrt{L-\tau}} d \tau+2 \sum_{n=1}^{\infty} \int_{0}^{L} \frac{i(\tau)}{\sqrt{L-\tau}} e^{\frac{-\beta^{2} n^{2}}{(L-\tau)}} d \tau .
$$

Observa-se que a partir da equação (4.6) o modelo RV pode ser utilizado para calcular o tempo de vida de baterias a partir do uso de cargas constantes ou de cargas variáveis. Neste contexto, considerando uma corrente de descarga constante no tempo $i(t)=I$, a equação (4.6) transformase em

$$
I=\frac{\alpha}{2 \sqrt{L}\left[1+2 \sum_{n=1}^{\infty}\left(e^{\frac{-\beta^{2} n^{2}}{L}}-\frac{\pi e^{\frac{-\beta^{2} n^{2}}{L}}}{\pi-1+\sqrt{1+\frac{\pi L}{\beta^{2} n^{2}}}}\right)\right]} .
$$

Entre as principais características do modelo RV está a fácil implementação necessitando os dados experimentais do problema real e de uma rotina inversa para a estimação de seus parâmetros empíricos. Analisando a equação (4.7) verifica-se que a mesma relaciona uma corrente de descarga $I$ como o tempo de vida $L$ e os parâmetros empíricos $\alpha$ e $\beta$ que dependem da bateria utilizada, e portanto precisam ser estimados. 


\subsection{Problema Inverso}

A análise de PIs constitui uma área multidisciplinar de pesquisa, com um grande número de aplicações em diferentes campos da ciência. Diferentes pesquisas acerca deste tema podem ser encontradas com abordagens variadas, mostrando que a análise de erros com uma rotina inversa pode ser satisfatória para a estimação de parâmetros com boa precisão [6, 12, 15, 24, 25, 26].

O problema inverso aqui considerado é formulado como um problema de otimização onde buscase minimizar o funcional de resíduos quadrados dado por

$$
Q(\vec{Z})=\sum_{i=1}^{N}\left(I_{e t_{i}}(\vec{Z})-I_{\exp _{i}}\right)^{2}=\left(\vec{E}_{i}\right)^{T} \vec{E}_{i}
$$

onde $\vec{E}_{i}=I_{\text {est }}(\vec{Z})-I_{\exp _{i}}, I_{\text {est }}(\vec{Z})$ é a corrente de descarga calculada pelo modelo e $I_{\text {est }}$ é a corrente de descarga experimental, $N$ é o número de medidas experimentais, $\vec{Z}$ é um vetor que contém os parâmetros que são estimados, dado por

$$
\vec{Z}=\{\alpha, \beta\}^{T} .
$$

Na próxima seção é apresentada a solução do PI considerando os métodos PRMe, PRM e MQ, assim como o resultado das simulações, comparação e análise.

\section{RESULTADOS DAS SIMULAÇÕES E ANÁLISE}

Nesta seção são apresentados os resultados das simulações do método de otimização PRMe aplicado ao modelo RV, para o cálculo dos seus parâmetros empíricos $\alpha$ e $\beta$, utilizando a ferramenta computacional MATLAB. Em seguida, são apresentados os resultados das simulações utilizando os métodos de otimização da literatura MQ e PRM, também aplicados ao modelo RV para o cálculo dos seus parâmetros empíricos $\alpha$ e $\beta$. Por fim, é realizado um estudo comparativo entre os resultados encontrados a fim de validar o método proposto.

Para a realização da tarefa de estimação de parâmetros do modelo RV é necessário um conjunto de dados reais da situação problema, que consiste no processo de descarregamento de uma bateria. Assim será utilizada a plataforma de teste, apresentada na Figura 2, com o objetivo de propiciar um ambiente completo para a realização de experimentos físicos envolvendo baterias. Maiores detalhes deste experimento podem ser encontrados em [16]. As baterias utilizadas nos experimentos são da tecnologia Li-Íon, modelo BL5F, presentes no telefone celular Nokia N95.

Para a realização dos ensaios experimentais foi adotado um padrão único em todos os experimentos, objetivando minimizar qualquer alteração no resultado final dos testes. Primeiramente, foram definidos os perfis de descarga seguindo o mesmo procedimento usado por Rakhmatov e Vrudhula [20] em que utilizaram um conjunto de dez correntes de descargas constantes. Sendo assim, os perfis usados neste trabalho são compostos por dez correntes de descargas constantes, medidas em miliampere $(m A)$. Os valores destas correntes foram distribuídos de maneira uniforme em relação a capacidade de carga total da bateria, abrangendo cargas baixas, médias e altas. 


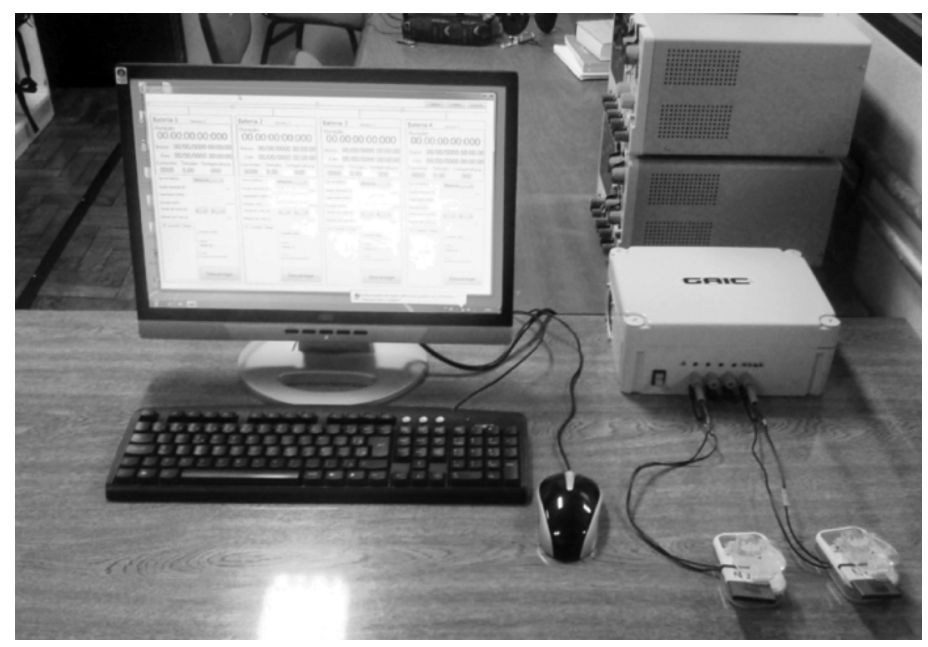

Figura 2: Plataforma de testes.

A metodologia adotada para a coleta de dados a partir da plataforma é descrita a seguir. Partiu-se da bateria completamente carregada (i.e., com uma voltagem de 4,2 volts), os experimentos foram divididos em dois momentos, o primeiro com dez perfis de descargas constantes apresentado na Tabela 1, utilizado para estimação dos parâmetros, e o segundo com três perfis de descargas constantes apresentado na Tabela 2, usado para a validação do modelo RV. Na primeira coluna de ambas as tabelas é apresentado o perfil de descarga ( $I$ ), e na segunda coluna o tempo de vida médio $\left(L_{m}\right)$. Para cada perfil de descarga considerado os ensaios foram repetidos dez vezes objetivando a obtenção de uma amostragem estatística satisfatória.

Tabela 1: Dados utilizados para estimação dos parâmetros do modelo RV [22].

\begin{tabular}{|c|c|}
\hline $\mathbf{I}(\mathbf{m A})$ & $L_{m}(\mathbf{m i n})$ \\
\hline 100 & 483,71 \\
\hline 150 & 325,67 \\
\hline 250 & 192,32 \\
\hline 350 & 136,25 \\
\hline 450 & 105,7 \\
\hline 550 & 85,8 \\
\hline 650 & 70,73 \\
\hline 750 & 60,74 \\
\hline 850 & 51,7 \\
\hline 950 & 46,7 \\
\hline
\end{tabular}

Inicialmente os parâmetros $\alpha$ e $\beta$ foram estimados pelo método PRMe, onde busca-se um conjunto de parâmetros que minimiza a equação (4.8), a partir do PD apresentado na equação (4.7). 
Tabela 2: Dados utilizados para validação do modelo RV [17].

\begin{tabular}{|c|c|}
\hline $\mathbf{I}(\mathbf{m A})$ & $L_{m}(\mathbf{m i n})$ \\
\hline 200 & 268,13 \\
\hline 500 & 268,13 \\
\hline 700 & 62,68 \\
\hline
\end{tabular}

Destaca-se que a equação do PD é complexa de resolver para $L$, então é considerado para os cálculos dos parâmetros o valor de $L_{m}$ obtido da plataforma de testes, sendo estimado o valor da corrente de descarga $I_{\text {est }}$, que é comparado com a corrente de descarga experimental $I_{\text {exp }}$ [20]. Observa-se que a equação (4.7) possui um parâmetro $n$, presente no somatório, que representa o número de dados experimentais obtidos da plataforma de testes. Neste trabalho há 10 dados experimentais, valor sugerido por Rakmatov e Vruhdula em [20].

Na Tabela 3 são apresentadas as iterações da execução do método PRMe. A metodologia de execução do método exige que inicialmente sejam atribuídos um valor inicial para cada parâmetro, neste trabalho são escolhidos $\alpha=1$ e $\beta=1$, mostrados na iteração 0 . O tamanho do intervalo foi definido pelo usuário, sendo aplicado um limite de $\pm 300 \%$, $\log$ o com $\rho=3$, com $q=100$ pontos. Cabe ressaltar que os parâmetros apresentados não foram os únicos a serem inspecionados. Durante a execução do método foram inspecionados 45 intervalos, mas apenas os 9 intervalos apresentados foram significantes, uma vez que contribuíram para a evolução do algoritmo, encontrando um conjunto de parâmetros com erro inferior ao conjunto anterior. O tempo de execução do método foi de aproximadamente 10 segundos. Na Tabela 4 são apresentados os valores para $\alpha$ e $\beta$ a partir do método PRMe.

Tabela 3: Resultados obtidos a partir da execução do método PRMe.

\begin{tabular}{|c|c|c|c|c|c|c|}
\hline Iteração & $\operatorname{Lim}_{\text {inf }} \alpha$ & $\alpha_{o t}$ & $\operatorname{Lim}_{\text {sup }} \alpha$ & $\operatorname{Lim}_{\text {inf }} \beta$ & $\beta_{o t}$ & $\operatorname{Lim}_{\text {sup }} \beta$ \\
\hline 0 & & 1 & & & 1 & \\
\hline 1 & -2 & 4 & 4 & -2 & 4 & 4 \\
\hline 2 & -8 & 16 & 16 & -8 & 16 & 16 \\
\hline 3 & -32 & 64 & 64 & -32 & 64 & 64 \\
\hline 4 & -128 & 256 & 256 & -128 & 256 & 256 \\
\hline 5 & -512 & 1024 & 1024 & -512 & 256 & 1024 \\
\hline 6 & -2048 & 4096 & 4096 & -512 & 256 & 1024 \\
\hline 7 & $-8192,00$ & 10674,42 & 16384,00 & $-512,00$ & 15,52 & 1024,00 \\
\hline 8 & $-21348,85$ & 19408,04 & 42697,70 & $-31,03$ & 4,70 & 62,06 \\
\hline 9 & $-38816,09$ & 18819,92 & 77632,18 & $-9,40$ & 4,84 & 18,81 \\
\hline
\end{tabular}


Tabela 4: Parâmetros do modelo RV, utilizando o método PRMe.

\begin{tabular}{|c|c|c|}
\hline Estimador & \multicolumn{2}{|c|}{ Procura Rede Melhorado } \\
\hline Parâmetro & $\alpha$ & $\beta$ \\
\hline Valor Estimado & 18820 & 4,84 \\
\hline
\end{tabular}

Após o cálculo dos parâmetros $\alpha$ e $\beta$ a partir do método de estimação de parâmetros PRMe, o tempo de vida da bateria foi calculados considerando o modelo RV, apresentado na equação (4.7), e as correntes de descargas constantes apresentadas na Tabela 2. Em seguida os tempos de vidas simulados pelo modelo $\left(L_{S}\right)$ foram comparados com os tempos de vida experimentais médios $\left(L_{m}\right)$ da Tabela 2. Na Tabela 5 são mostrados os resultados desta comparação.

Tabela 5: Validação do modelo RV utilizando o método PRMe para estimação dos parâmetros.

\begin{tabular}{|c|c|c|c|c|}
\hline Perfil de Descarga $(\mathrm{mA})$ & $L_{m}(\min )$ & $L_{s}(\mathrm{~min})$ & Erro & Erro (\%) \\
\hline 200 & 268,13 & 247,22 & 20,90 & 7,79 \\
\hline 500 & 98,23 & 94,33 & 3,89 & 3,96 \\
\hline 700 & 62,68 & 65,22 & 2,54 & 4,05 \\
\hline \multicolumn{4}{|c}{ Valor do Erro Médio(\%) } \\
\hline
\end{tabular}

Na validação do modelo, é possível notar que com cargas menores (e.g. $200 \mathrm{~mA}$ ) o modelo demonstrou menor precisão em comparação com cargas altas de $700 \mathrm{~mA}$, este fato se dá devido a correntes de descargas de baixa intensidade terem um tempo maior de descarga da bateria, deixando assim o processo sujeito as não linearidades do sistema. Neste cenário de simulação o modelo RV obteve um erro médio de 5,27\%.

A seguir são apresentados na Tabela 6, os valores dos parâmetros $\alpha$ e $\beta$ do modelo RV, a partir da utilização do método MQ, observa-se que o tempo de execução deste método é instantâneo [17, $20,21,22]$.

Tabela 6: Parâmetros do modelo RV, utilizando o método MQ.

\begin{tabular}{|c|c|c|}
\hline Estimador & \multicolumn{2}{|c|}{ Mínimos Quadrados } \\
\hline Parâmetro & $\alpha$ & $\beta$ \\
\hline Valor Estimado & 19993 & 4,5 \\
\hline
\end{tabular}

De posse dos parâmetros $\alpha$ e $\beta$ a partir do método MQ, o tempo de vida da bateria foi calculado considerando o modelo RV e os dados para as correntes de descargas apresentadas na Tabela 2. Em seguida, os tempos de vidas simulados pelo modelo $\left(L_{S}\right)$ foram comparados com os tempos de vida experimentais médios $\left(L_{m}\right)$ da Tabela 2. Na Tabela 7 são apresentados os resultados desta comparação. 
Tabela 7: Validação do modelo RV utilizando o método MQ para estimação dos parâmetros.

\begin{tabular}{|c|c|c|c|c|}
\hline Perfil de Descarga $(\mathrm{mA})$ & $L_{m}(\mathrm{~min})$ & $L_{s}(\mathrm{~min})$ & Erro & Erro (\%) \\
\hline 200 & 268,13 & 245 & 23,13 & 8,62 \\
\hline 500 & 98,23 & 94 & 4,23 & 4,30 \\
\hline 700 & 62,68 & 65,33 & 2,65 & 4,23 \\
\hline \multicolumn{4}{|c}{ Valor do Erro Médio (\%) } \\
\hline
\end{tabular}

Pode-se observar que o erro diminui a medida que a corrente aumenta. Para o perfil $200 \mathrm{~mA}$, que é a menor corrente de descarga, foi encontrada a maior diferença entre os tempos, ou seja, $8,62 \%$, já para o perfil $700 m A$, que corresponde a maior corrente de descarga, a diferença entre os tempos é de 4,23\%. Estas diferenças estão relacionadas aos efeitos não lineares que ocorrem durante uma corrente de descarga, que atuam com maior frequência em correntes de descargas baixas. Neste cenário de simulação o modelo RV obteve um erro médio de 5,72\%.

Por fim, são apresentados os resultados das simulações para a obtenção dos parâmetros $\alpha$ e $\beta$ do modelo RV, a partir da utilização do método PRM, onde busca-se um conjunto de parâmetros que minimiza a equação (4.8), a partir do PD apresentado na equação (4.7). Para a estimação dos parâmetros foram utilizados os dados apresentados na Tabela 1. A metodologia de execução deste método exige que inicialmente sejam inseridos vetores com intervalos mínimos e máximos que possam conter o valor ótimo dos parâmetros a serem estimados. Esta tarefa de encontrar os intervalos válidos é uma característica empírica do método, uma vez que sem o conhecimento algum do modelo esta busca pode não chegar a um resultado satisfatório. Com isto, sugere-se que inicialmente seja feita uma busca em um intervalo com dimensões grandes, para então se aproximar do valor ótimo com intervalos cada vez menores.

Na Tabela 8 é apresentado o processo de estimação de parâmetros pelo método PRM, onde estão especificados os intervalos selecionados em cada procura realizada, bem como o melhor valor encontrado em cada intervalo. Observa-se que a partir da décima execução o algoritmo convergiu para os valores de $\alpha$ e $\beta$, ao todo foram realizadas 13 execuções do algoritmo. Cabe ressaltar que o algoritmo poderia continuar a ser executado, buscando um refinamento do valor, mas isso não necessariamente se converteria em um valor com menor erro requerido.

Verifica-se também que a metodologia do algoritmo exige que o usuário defina um número de divisões dos intervalos propostos, assim durante os testes realizados para a implementação do algoritmo o valor de 100 divisões demonstrou ter a melhor relação tempo versus precisão. Um valor abaixo de 100 o método perdeu precisão, em contrapartida com um valor acima acarretou um aumento no tempo de execução do algoritmo, sem aumentar significativamente a precisão. Por outro lado, observa-se que as 5 primeiras execuções do método, por se tratar de intervalos considerados grandes, utilizou-se uma divisão de 1000 pontos, isso ocorreu para que o espaçamento entre pontos não fosse tão grande, deixando assim de analisar uma quantidade grande de valores. O tempo total das execuções deste método considerando a análise necessária entre as execuções foi de aproximadamente 20 horas. Na Tabela 9 são apresentados os valores para $\alpha$ e $\beta$ a partir do método PRM. 
Tabela 8: Resultados da execução do método PRM.

\begin{tabular}{|c|c|c|c|c|c|c|c|}
\hline Iteração & Lim $_{\text {inf }} \alpha$ & $\alpha_{\text {ot }}$ & $\operatorname{Lim}_{\text {sup }} \alpha$ & $\operatorname{Lim}_{\text {inf }} \beta$ & $\beta_{\text {ot }}$ & $\operatorname{Lim}_{\text {sup }} \beta$ & Tempo \\
\hline 1 & -100000 & 10800 & 100000 & -100000 & 10800 & 100000 & 600 \\
\hline 2 & 0 & 10830 & 30000 & 0 & 10830 & 30000 & 59 \\
\hline 3 & 0 & 9990 & 10000 & 0 & 29970 & 30000 & 62 \\
\hline 4 & 0 & 9990 & 10000 & 0 & 999 & 1000 & 68 \\
\hline 5 & 0 & 9990 & 10000 & 0 & 99,9 & 100 & 170 \\
\hline 6 & 0 & 19980 & 20000 & 0 & 99,9 & 100 & 92 \\
\hline 7 & 0 & 19920 & 30000 & 0 & 6,64 & 10 & 68 \\
\hline 8 & 10000 & 20000 & 30000 & 3 & 6,5 & 10 & 69 \\
\hline 9 & 15000 & 19650 & 30000 & 0 & 3,1 & 10 & 1,2 \\
\hline 10 & 15000 & 20000 & 25000 & 0 & 5 & 10 & 1,2 \\
\hline 11 & 18000 & 19920 & 22000 & 3 & 4,92 & 7 & 1,2 \\
\hline 12 & 19000 & 19920 & 21000 & 4 & 4,92 & 6 & 1,2 \\
\hline 13 & 19500 & 19990 & 20500 & 4,5 & 4,99 & 5,5 & 1,2 \\
\hline
\end{tabular}

Tabela 9: Parâmetros do modelo RV, utilizando o método PRM.

\begin{tabular}{|c|c|c|}
\hline Estimador & \multicolumn{2}{|c|}{ Procura Rede Modificado } \\
\hline Parâmetro & $\alpha$ & $\beta$ \\
\hline Valor Estimado & 19990 & 4,99 \\
\hline
\end{tabular}

Após o cálculo dos parâmetros $\alpha$ e $\beta$ a partir do método de estimação de parâmetros PRM, o tempo de vida da bateria foi calculado considerando o modelo RV e os dados apresentados na Tabela 2. Em seguida os tempos de vidas simulados pelo modelo $\left(L_{S}\right)$ foram comparados com os tempos de vida experimentais médios $\left(L_{m}\right)$ da Tabela 2. Na Tabela 10 são mostrados os resultados desta comparação.

Tabela 10: Validação do modelo RV utilizando o método PRM para estimação dos parâmetros.

\begin{tabular}{|c|c|c|c|c|}
\hline Perfil de Descarga $(\mathrm{mA})$ & $L_{e}(\mathrm{~min})$ & $L_{c}(\mathrm{~min})$ & Erro & Erro $(\%)$ \\
\hline 200 & 268,13 & 317,92 & 49,79 & 18,57 \\
\hline 500 & 98,23 & 112,23 & 14 & 14,26 \\
\hline 700 & 62,68 & 66,69 & 4,01 & 6,4 \\
\hline \multicolumn{4}{|c|}{ Valor do Erro Médio(\%) } & 6,53 \\
\hline
\end{tabular}

Analisando os erros obtido na validação do modelo RV, é possível notar que com cargas menores (e.g. $200 \mathrm{~mA}$ ) o modelo demonstrou menor precisão em comparação com cargas altas de 700 $m A$, este fato se dá devido a cargas de baixa intensidade terem um tempo maior de descarga 
da bateria, deixando assim o processo sujeito as não linearidades do sistema. Neste cenário de simulação o modelo RV obteve um erro médio de $6,53 \%$.

Após os resultados das simulações considerando os métodos de otimização PRMe, MQ e PRM verificou-se que o método PRMe demonstrou ser um método de implementação acessível e boa velocidade de execução, em relação aos outros métodos simulados. A acessibilidade em relação a implementação consiste no seu funcionamento, onde a partir de 10 perfis de descargas experimentais da bateria e da função objetivo foi realizada a estimação de parâmetros do modelo RV. O algoritmo do método, a partir de uma valor inicial para os parâmetros, realiza a criação de redes e busca nestas redes o melhor valor para os parâmetros, caso o valor encontrado não satisfaça o erro requerido, o algoritmo, a partir dos valores obtidos para os parâmetros, cria uma nova rede e realiza uma nova busca. Este processo, que é feito de forma exaustiva, possibilita convergência do método para a melhor rede, de modo que se obtenha o melhor valor para os parâmetros procurados.

Realizando a comparação entre os resultados encontrados a partir do método PRMe e do método MQ percebe-se que em relação a validação do modelo RV os dois métodos possuem uma diferença muito pequena em relação a precisão, ou seja, o primeiro apresentou um erro médio de $5,27 \%$ e o segundo um erro médio de 5,72\%. Isto demonstra que embora de metodologias diferente na estimação, os dois métodos apresentam valores para os parâmetros próximos. Porém, destaca-se que o método PRMe possui maior simplicidade na implementação do que o método MQ, pois utiliza um algoritmo de buscas a partir da função objetivo executada de forma exaustiva necessitando apenas de dados de entrada e saída obtidos através de experimentos realizados em uma plataforma de testes.

Realizando a comparação entre o método PRMe e o método PRM nota-se uma diferença percentual na validação do modelo RV, onde o método PRMe demonstrou possuir uma maior acurácia, com erro médio de 5,27\% em relação ao método PRM, com erro médio de 6,53\%. Esta acurácia ocorre devido a diferença de metodologia de busca dos parâmetros. O método PRM realiza buscas em redes sugeridas a partir de observações dos perfis de descarga e posteriormente uma redução na amplitude do intervalo, baseado em decisões do usuário do método. Enquanto que o método PRMe realizou buscas em 40 diferentes redes, porém o algoritmo evoluiu em relação aos intervalos das redes, sem a interferência do usuário, até convergir para um dado valor dos parâmetros. Ainda que o método PRMe realize mais buscas, o tempo de execução não foi prejudicado, enquanto o método PRMe demorou 10 segundos para executar o seu algoritmo, o método PRM demorou em torno de 20 horas.

\section{CONCLUSÕES}

Neste trabalho foi realizada a proposição de um novo método de otimização para estimação de parâmetros, denominado método da Procura em Rede Melhorado, aplicado ao modelo RV, que é um dos modelos mais acurado da literatura técnica para a predição do tempo de vida de baterias usadas em dispositivos móveis. A principal característica deste método é a realização da esti- 
mação de parâmetros utilizando dados experimentais e a função objetivo do modelo. Observa-se que a busca dos parâmetros é realizada de forma simples e dinâmica.

O novo método foi avaliado considerando a seguinte metodologia: inicialmente os parâmetros $\alpha$ e $\beta$ do modelo RV foram estimados considerando os estimadores PRMe, MQ e PRM, bem como os dados experimentais obtidos a partir de uma plataforma de testes, considerando uma bateria de Li-Íon, usada no celular Nokia 95, modelo BL5F; em seguida o modelo RV foi validado para cada conjunto de parâmetros e os dados simulados a partir do modelo foram comparados com outro conjunto de dados experimentais obtidos da plataforma; e por fim foi realizada uma análise comparativa entre os resultados obtidos.

A partir da análise comparativa entre os resultados das simulações do modelo RV considerando do método PRMe, e do método MQ, observou-se que dois métodos possuem uma diferença muito pequena em relação a precisão, ou seja, o primeiro apresentou um erro médio de 5,27\% e o segundo um erro médio de 5,72\%. Embora ambos apresentem a precisão próxima e pouca diferença no tempo de execução, ressalta-se que o método PRMe possui maior simplicidade na implementação do algoritmo do que o método MQ, visto que o método PRMe utiliza um algoritmo de buscas a partir da função objetivo executada de forma exaustiva necessitando apenas de dados de entrada e saída obtidos através de experimentos realizados em uma plataforma de testes. Realizando a comparação entre o método PRMe e o método PRM nota-se uma diferença percentual na validação do modelo RV, onde o método PRMe demonstrou possuir uma maior acurácia, com erro médio de 5,27\% em relação ao método PRM, com erro médio de 6,53\%, bem como uma grande diferença no tempo de execução, enquanto o método PRM demora em torno de 20 h para finalizar a execução do programa na busca pelos parâmetros, o método PRMe é quase instantâneo.

Considerando os resultados encontrados satisfatórios conclui-se que o método PRMe possui fácil implementação, boa velocidade de execução na estimação dos parâmetros, bem como boa precisão do modelo, sendo assim ele pode ser recomendado para estimação de parâmetros em outras áreas da ciências.

Como trabalhos futuros, sugere-se que o estimador seja utilizado em outros modelos matemáticos objetivando-se concluir a robustez do método, e posteriormente a comparação com métodos de estimação indicados a estes modelos. Também se faz necessária uma investigação em relação a linguagem de programação utilizada, isto pode representar um ganho em relação ao tempo e uma amplitude na criação de redes maiores. Ainda sugere-se estender este trabalho realizando uma análise estatística dos resultados encontrados.

\section{AGRADECIMENTOS}

Os autores agradecem ao apoio financeiro da Fundação de Amparo à Pesquisa do Rio Grande do Sul (FAPERGS) a partir do Edital 02/2011 (PQG). 


\begin{abstract}
This paper presents the proposition of a new optimization method called Enhanced Network Search, which is an extension of the method Modified Network Search to calculate the empirical parameters of the Rakhmatov and Vrudhula model to predict the mobile devices battery lifetime. The new method was evaluated according to the following methodology, at first empirical parameters were computed considering the optimization methods of Improved Network Search, Modified Network Search and Least Squares, as well as the experimental data obtained from a testbed, considering a Lhition-Ion battery, model BL5F, used in the Nokia N95 cell fone. In a second moment, the Vrudhula and Rakhmatov model was validated for each set of parameters obtained, and the simulated data from the model were compared with a set of experimental data. From simulations results was performed a comparative analysis and was found that with the application of the method Enhanced Search Network in the estimation of parameters of the Rakhmatov and Vrudhula model was possible to obtain an easy and intuitive implementation, improving the results obtained in the model accuracy, as well as preserves the runtime.
\end{abstract}

Keywords: estimation of parameters, Enhanced Network Search, model RV.

\title{
REFERÊNCIAS
}

[1] L. A. Aguirre. "Introdução à Identificação de Sistemas: Técnicas Lineares e Não-Lineares Aplicadas a Sistemas Reais", Editora UFMG, Belo Horizonte (2007).

[2] A.L.J. Bihain, L.D.T. Câmara \& A.J. da Silva Neto. Avaliação da Rotina Inversa R2W na Estimação de Parâmetros de Transferência de Massa no Processo de Adsorção de Glicose e Frutose. Tendências em Matemática Aplicada e Computacional, 2(1) (2001), 1-12.

[3] P.A.P. Borges, M.T. Vione \& A. Cervi. "Determinação dos Coeficientes do Modelo de Van Genuchten Empregando o Problema Inverso". Anais do VIII Encontro Regional de Matemática Aplicada e Computacional, Pelotas (2008).

[4] H.F. Campos Velho. "Problemas inversos: conceitos básicos e aplicações". Anais do Encontro de Modelagem Computacional. Mini-curso, (2001), 63-79.

[5] A. Cervi. "Determinação dos Parâmetros da Equação Característica de Solos Através de Técnicas de Solução de Problemas Inversos com Base em Dados de Evaporação", Dissertação de Mestrado, UNIJUÍ (2009).

[6] A. de Cezaro. "Problemas Inversos: Uma Introdução". SBM, Colóquio de Matemática da Região Sul, 1 (2010).

[7] M. Chen \& G.A. Rincón-Mora. Accurate electrical battery model capable of predicting runtime and I-V performance. IEEE Transactions on Energy Conversion, 21(2) (2006).

[8] C. Chiasserini \& R. Rao. A Model for Battery Pulsed Discharge with Recovery Effect, Proceedings of the Wireless Communications and Networking Conference, (1999), 636-639.

[9] H.W. Engl, M. Hanke \& A. Neubauer. "Regularization of Inverse Problems: Mathematics and its Applications", Mathematics and its Applications Series, Kluwer Academic Plubishers (1996). 
[10] M. Jongerden \& B. Haverkort. Battery Modeling, Technical Report in Faculty Electrical Engineering, Mathematics and Computer Science, January (2008).

[11] K. Lahiri, A. Raghunathan, S. Dey \& D. Panigrahi. Battery-driven system design: a new frontier in low power design. Chemical Engineering Science (2002).

[12] L. Ljung. "System Identification Theory for the User", Prentice Hall, USA (1999).

[13] T.L. Martin. "Balancing Batteries, Power, and Performance: System Issues in CPU Speed-Setting for Mobile Computing”. Master's Thesis, Carnegie Mellon Unerversity (1999).

[14] N.M. McCormick. Inverse problems: methods and applications. TEMA, Tendências em Matemática Aplicada e Computacional, 13(3) (2012), 277-289.

[15] M.I. Mesa, O.L. Santiago, F.H. Fernández Accurate, D.C. Rodríguez, A.J. da Silva Neto \& L.D.T. Câmara. An approach to parameters estimation of a chromatography model using a clustering genetic algorithm based inverse model. Soft Comput., 15(5) (2011), 963-973.

[16] H.B. Nonemacher, L. Minelli, P.S. Sausen \& A. Sausen. Desenvolvimento de um testbed para avaliação de modelos matemáticos utilizados na predição do tempo de vida das baterias, XXIV CRICTE, Congresso Regional de Iniciação Científica e Tecnológica em Engenharia, Rio Grande, RS (2010).

[17] A.V. Oliveira. "Análise Comparativa de Metodologias de Estimação de Parâmetros Aplicada a Modelos Analíticos Utilizados na Predição do Tempo de Vida de uma Bateria”, Dissertação de Mestrado, UNIJUÍ, Ijuí, RS (2012).

[18] C.M.D. Porciuncula. "Aplicação de Modelos Elétricos de Bateria na Predição do Tempo de Vida de Dispositivos Móveis”, Dissertação de Mestrado, UNIJUÍ, Ijuí, RS (2012).

[19] C.M.D. Porciuncula, A. Oliveira, P.S. Sausen \& A. Sausen. Avaliação Comparativa entre o Modelo Elétrico Battery e os Modelos Analíticos Linear e Lei de Peukert. Revista Brasileira de Computação Aplicada, 4(1) (2012), 71-80.

[20] D. Rakhmatov \& S. Vrudhula. An Analytical High-Level Battery Model for Use in Energy Management of Portable Electronic Systems. Proceedings of the 2001 IEEE/ACM International Conference on Computer-Aided Design, (2001), 488-493.

[21] P. Sausen. "Gerenciamento Integrado de Energia e Controle de Topologia em Redes de Sensores Sem Fio", Tese de Doutorado, UFCG, Campina Grande, PB (2008).

[22] K.K. Schneider. "Modelos Analíticos na Predição do Tempo de Vida de Baterias Utilizadas em Dispositivos Móveis”. Dissertação de Mestrado, UNIJUÍ, Ijuí, RS (2011).

[23] K.K. Schneider, P.S. Sausen \& A. Sausen. Análise comparativa do tempo de vida de baterias em dispositivos móveis a partir da utilização de modelos analíticos. TEMA, Tendências Matemática Aplicada e Computacional, 12(1) (2011), 43-54.

[24] A.J. Silva Neto. "Problemas Inversos: Conceitos Fundamentais e Aplicações", Ed. UERJ, Rio de Janeiro, RJ (2005).

[25] A.J. Silva Neto \& J.C. Becceneri. "Técnicas de Inteligência Computacional Inspiradas na Natureza: Aplicações em Problemas Inversos em Transferência Radiativa”. Notas em Matemática Aplicada, 41 (2009).

[26] A. Tarantola. "Inverse Problem Theory and Methods for Model Parameter Estimation", SIAM, Paris, France (2005). 